\title{
Michelson interferometer application for measuring films and glass refractive index
}

\section{Felipe L. P. Ferreira (IC), Luis F. de Avila (PQ).}

\section{Abstract}

This project aims to study and characterization of the refractive index of thin films and glasses by using a Michelson interferometer.

Key words: refractive index, Michelson interferometer, instrumentation.

\section{Introduction}

The knowledge of the refractive index of materials is important to the development of applications. Thus, have been developed several refractometry techniques for measuring the refractive index. Among the refractometry techniques we can cite the Abeles method, the ellipsometry, a hollow prism that allows you to store liquid to be measured the refractive index, etc. Many interferometric methods for determining the refractive index of materials have also been developed [1] [2] . They can use the Rayleigh refractometer, Mach-Zehnder, Jamin, Fabry-Perot and Michelson. The Michelson interferometer can be used for refractive index and thickness measurements of glasses and thin films. These measurements can be performed with great precision, since interferometry technique utilizes the interference nanometers wavelength .

In this work we assemble a Michelson interferometer for measuring the refractive index of erbium doped glasses synthesized in UNESP Araraquara with potential application in the fabrication of photonic crystals. The measurement of refractive index is obtained by rotating the sample placed in an interferometer arm, creating an optical path variation, and hence the movement of the interference fringes. The expression for the refractive index in terms of the number of fringes collapsing and the angle by which the sample has been rotated is given by:

$$
n=\frac{(2 t-N \lambda)(1-\cos i)}{2 t(1-\cos i)-N \lambda}
$$

Where $\mathrm{N}$ is the number of fringes, $\mathrm{t}$ the thickness of the material, $\lambda$ the laser wavelength and $i$ the angle by which the sample has been rotated.

\section{Results and Discussion}

The Figure 1 Shows schematic of the Michelson interferometer and a photo of the setup constructed. To determine the angle at which the sample was rotated, an automated base has been developed. The base is fixed in one of the interferometer arms and consists of a stepping motor fixed on the table and the sample holder is integrated to the motor allowing rotation of the glass sample into the table plane. To rotate the sample, was used one microcontrolled circuit with PIC18F4550, who commands the stepper motor by sending pulses to a circuit with 4 transistors that determines the rotation angle.

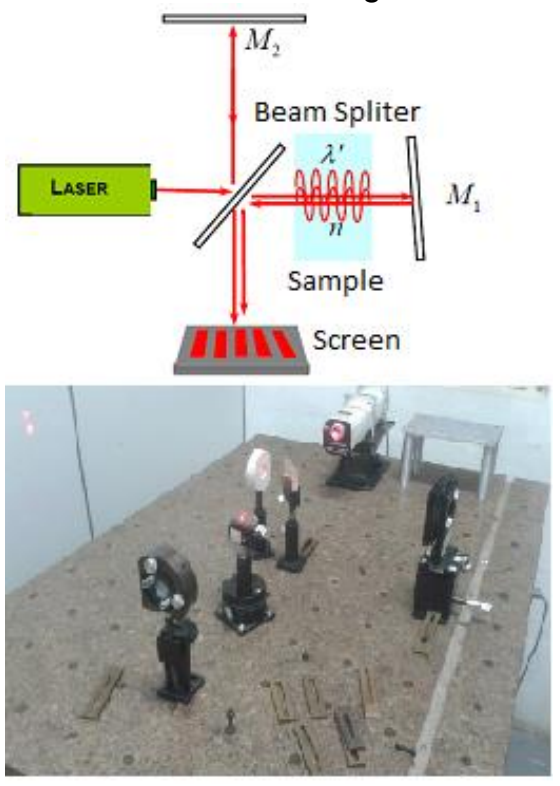

Figure 1 - Michelson interferometer setup.

\section{Conclusions}

In this work we study a Milchelson interferometer applied to measure the refractive index of glasses and films. It was also developed an automated support placed in one of the interferometer arms allowing the rotation of the sample. The next step is to measure the refractive index of the samples and make adjustments on acquisition system.

\section{Acknowledgement}

The authors thank the Conselho Nacional de Desenvolvimento Científico e tecnológico (CNPq) and the Serviço de Apoio ao Estudante (SAE) of Pró Reitoria de Graduação of UNICAMP (PRG) for the financial support.

\footnotetext{
Carlos R. A. Lima, "Roteiro Experimental de Física IV" http://www.fisica.ufjf.br/ cralima/index_arquivos/LabFisIV/LabfisI V.pdf Acessado em: 09/04/2014

Iyer, Deepak N., "A Michelson interferometric technique for measuring refractive index of sodium zinc tellurite glasses" (2006). http://preserve.lehigh.edu/cgi/viewcontent.cgi?article=1954\&context =etd Acessado em: 09/04/2014
} 